\title{
SPATIAL DATA INFRASTRUCTURE (SDI) IN OMAN AND QATAR - CURRENT STATUS AND PERSPECTIVES
}

\author{
Khalid Al Kalbani ${ }^{1,}$, Alias Abdul Rahman ${ }^{1}$, Ammar Abulibdeh $^{2}$, Mohammed J. Sani ${ }^{1}$, Hanis Rashidan ${ }^{1}$ \\ 13D GIS Research Lab, Faculty of Built Environment and Surveying, Universiti Teknologi Malaysia, Johor, Malaysia. \\ ${ }^{2}$ Department of Humanities, Qatar University, Doha, Qatar. \\ *kulaib2020@gmail.com, alias@utm.my,mjsani71@gmail.com, mhanis27@gmail.com \\ aabulibdeh@qu.edu.qa
}

Commission 4, WG 7

KEYWORDS: SDI, 3D SDI, BIM, CityGML, 3D City Model.

\begin{abstract}
Urban infrastructure deals with various objects, such as multi-floor buildings, underground utilities, and other typical two-dimensional (2D) geographic information system (GIS) objects. Currently, Oman and Qatar authorities attempt to manage and handle all these spatial objects and features via spatial data infrastructure (SDI). Qatar has a long tradition of applying SDI, while Oman has made rapid progress in building SDI according to the current practices in this field. Governments in both countries work toward smart cities and e-government to meet the fourth industrial revolution requirements. Current literature, indicate that SDI could be considered a major building block for such smart initiatives in the near future. This paper describes the status and challenges of developing SDI (including 3D SDI) for Oman and Qatar. We anticipated these 2D and 3D SDI initiatives would eventually provide future direction for the smart city and e-government. The current status and challenges form major discussions of this paper. It also discusses 3D SDI development. This paper ends by describing a framework for implementing the 3D SDI. The outcomes could significantly improve the awareness for developing 3D SDI in Oman and Qatar.
\end{abstract}

\section{INTRODUCTION}

GIS provides enormous benefits in managing our surroundings and environment where well-established systems either local or/and national were in placed as reported by many practitioners and researchers in many countries (UN GGIM 2011 and 2013). UN GGIM is an entity dealing with various aspects of spatial data management in UN member states. However, each member state has a different level of achievement. One of the goals of this UN GGIM entity is to learn from each other and eventually well managed geospatial data and the related infrastructure (Cheung, 2011). Here, one of the main discussions at this level of the forum was spatial data infrastructure (SDI). Many countries are now developing SDI and some with advanced services where data sharing and applications in placed and reachable by users. Today, we have seen some nations go beyond the traditional working toward the 3D version of the SDI. Attempts are being made by Australia, Netherlands, Finland, Germany as reported by Abbas et al (2019) and also by Chaturvedi and Kolbe (2019). Countries like Oman and Qatar are also at the stage of developing 3D SDI. It is the aim of this paper to describe the development of 2D SDI and also how researchers and authorities of these two countries engage and address the initiative on 3D SDI. Khalid Al Kalbani spent several years at Universiti Teknologi Malaysia (UTM) for his research on issues and challenges of developing 3D SDI for Oman based on 3D city model with respect to CityGML data exchange format. Whereas Ammar Abulibdeh investigating the 2D SDI in general and attempts to extend the current state of the SDI to 3D for Qatar. Currently, Oman and Qatar have made noticeable progress in SDI and e-government but not yet in 3D SDI. The remaining of this paper describes other aspects of SDI of these two countries in the following order.

This paper describes the state-of-the-art of the SDI in Oman and Qatar in Section 2, the development attempts by these two member states in Section 3 as far 2D and 3D SDI. We also attempt to highlight several issues and challenges in establishing such SDI initiatives in Section 4, and finally conclude and offered several recommendations on how these important aspects of GIS could be addressed by the authorities.

\section{THE CURRENT STATUS OF SDI - OMAN AND QATAR}

\subsection{Oman}

Oman government has established its national SDI since 2014 to standardise the geospatial activities and business at the national level. The institutional structure for Oman National Spatial Data Infrastructure (ONSDI) consists of a group of supervisory and executive institutions and authorities. The ONSDI has gained success in the efforts of homogenising the geospatial data and has a standardised geo-portal. Also, it has made noticeable progress in managing the 2D geo-dataset of the government sectors (NCSI, 2017b, 2017a). ONSDI has built it's National Geodatabase (NGDB) to be the master host for all Oman spatial data based on assessing the geospatial community and stakeholders' requirements in Oman. The NGDB was built based on international standards (OGC and ISO) adapted to the Omani requirement (NCSI, 2017b, 2017a). ONSDI, in coordination with National Survey Authority (NSA), has published the Unified National Basemap (UNB) and launched it in the National Centre for Statistical Information (NCSI) portal for (Public, private) users, as shown in Figure 1. Oman geospatial community and stakeholders have been able to use the UNB dataset for their core business and synchronise online their geodatabase with the current Oman data version all the time. Additionally, the ONSDI provides geo-dataset through different services (GIS Web and phone applications) for public and private stakeholders according to the permitted level. 


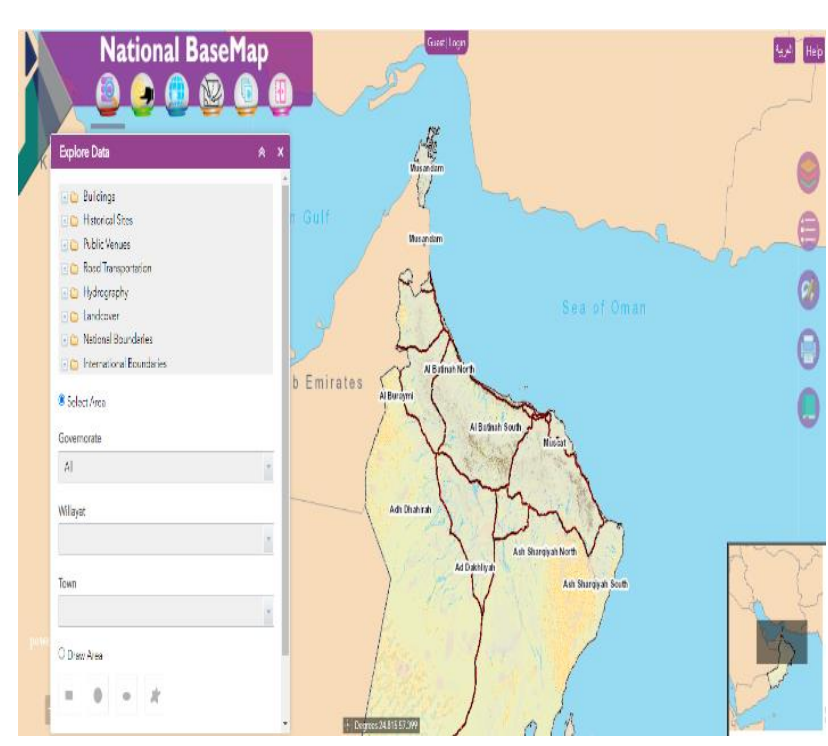

Figure 1. The Unified National Basemap (UNB).

In Oman geospatial community, the 3D spatial data is limited. As clarified by NCSI (2017a, 2017b), the majority of the Oman main sectors have preserved the flow of spatial activity in $2 \mathrm{D}$ and $2.5 \mathrm{D}$ spatial data. Generally, the $2.5 \mathrm{D}$ spatial data are used in Oman (public and private sectors) to map and analyse the geomorphological, hydrological, geological data and mineral land classification. In contrast, the 3D spatial data are used for some applications and projects like 3D simulation of the safety and security programs in oil installations (Das et al., 2017), including creating 3D models in CAD software for viewing the planning and transportation projects before establishing them. No clear vision or legislation encourages the Oman sectors to invest in 3D spatial data. While the ONSDI and other stakeholders have started using 2D and 2.5D spatial data, the 3D spatial data implementation seems uncertain. Knowing the importance and benefits of the 3D spatial data is not sufficient in the Oman geospatial community.

Oman is one of the countries which have a complex urban infrastructure, as shown in Figure 2. Hence, decision-makers in Omani municipalities require 3D SDI to manage complex data structures. The 3D SDI is considered a significant investment for future applications, such as 3D cadastral, 3D smart city and flood risk assessment. Oman's government has succeeded in taking the basic steps to include SDI as a mechanism for organising and managing geospatial data according to international standards. Nevertheless, there are deficiencies in assessing the challenges of geospatial data implementation and the future needs of stakeholders in terms of 3D geospatial data and its applications.

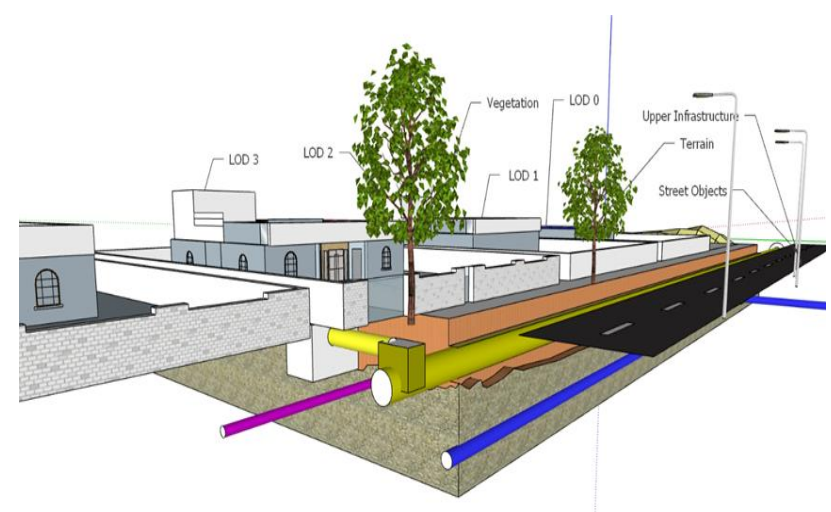

Figure 2. The complexity of urban infrastructure in Oman.

\subsection{Qatar}

The state of Qatar has witnessed rapid population growth, economic development, and rapid urbanism during the past three decades driven by the abundant wealth of oil and gas. Consequently, there was a pressing need for an assessment of the geospatial setup of the country. In 1992, the State of Qatar has established the Centre for Geographic Information Systems (CGIS) to create, maintain, and update Qatar's Geodetic Network, National GIS and GPS standards and specifications, high-speed GIS data sharing network (GISnet), and Topographic Database. The GIS information was nationwide between more than 90 government and semi-government agencies integrated within this system. The objective is to enhance inter-agency coordination and to avoid duplication of efforts and data redundancy. This was associated with the emerging international directions and initiatives such as NSDI, UN-GGIM, trained and motivated manpower data updating, trends in data concepts, data security, and demand for processed data for a new GIS strategy known as Qatar National Geospatial Information Data Infrastructure (QNGDI). The purpose of creating the QNGDI was to develop a state of the art national geospatial infrastructure that guarantees the availability of timely, reliable, and secured geospatial information and services to all stakeholders involved in the process of nation-building and to support social, economic, and human development towards a sustainable society.

The CGIS implemented large-scale projects such as the Qatar Area Referencing System (QARS) "Inwani" and the National Mapping project (NM). The QARS project aims to help in the development of detailed area planning and addressing, and including the address plates and their data into the project's digital mapping database as shown in Figure 3. The NM project aimed to update the digital topographic database as shown in Figure 4. The project achieved creating Digital Aerial Oblique Color Photography 4 - Directional $-10 \mathrm{~cm}$ resolution for 3D city model facades for the main cities in Qatar (i.e., Al Doha, Al Khor and Al Wakra). Furthermore, the project created Aerial LiDAR having $5 \mathrm{~cm}$ vertical accuracy with 4 points per sq. meter point cloud and Aerial Triangulation for all urban areas including the coastline. The project generated a 3D City Model with absolute (30 cm horizontal and $25 \mathrm{~cm}$ vertical) accuracy with LoD2 and LoD3 for the same cities. 


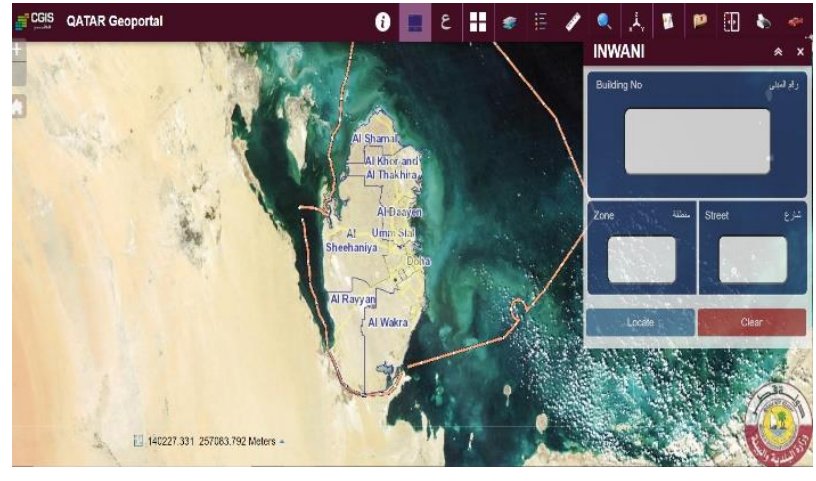

Figure 3. QATAR Geoportal

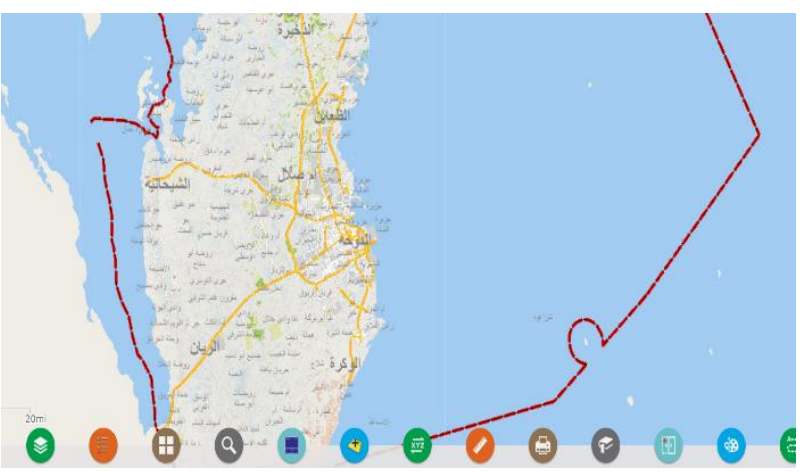

Figure 4. National Mapping project

\section{DEVELOPING 3D SDI BASED ON 3D CITY MODEL}

In this section, several important aspects of developing 3D SDI will be described with the following details focusing on spatial data, standards, data services.

\subsection{Spatial data and file formats}

In order to establish the upcoming 3D SDI in Oman and Qatar, the system should be able to facilitate with a wide range of $2 \mathrm{D}$ and 3D spatial data sources and file formats as the following:

Cadastral Data: This data is considered essential for surfaces and sub-surfaces components of spatial objects. Cadastral data normally contains ownership information, land parcel (boundaries and boundary marks, lot numbers, area), and access roads. There are different types of cadastral data, such as land parcels, footprints of buildings, footpaths, ownership information. Therefore, the $2 \mathrm{D}$ and $3 \mathrm{D}$ cadastral data is an important element for generating spatial objects models at LoD0 LoD4.

Terrain Data: Terrain data or digital terrain model (DTM) easily available within the agency (Oman SDI) in various formats such as XYZ, GeoTIFF, IMG (binary image), and contours data, point clouds are important data to create the surface structure (terrain model) for the 3D city model.

LiDAR Data: LiDAR is one of the popular 3D data sources and is quite costly. However, this category of data is hardly available in Oman. This data type has been utilised by several national mapping agencies (outside Oman) for 3D buildings generation (for 3D city models purposes). Other spatial objects such as trees, power lines structures, and terrain can be extracted from the point cloud for other mapping purposes.

Satellite, Airborne and Terrestrial Datasets: Datasets such as aerial photographs (including oblique images), satellite imageries, and 360-degree panoramic images are examples of the important spatial data for developing SDI. The availability of these kinds of datasets would improve the robustness of SDI services in Oman (and Qatar).

Architecture Engineering and Construction (AEC) / Facility Management (FM): These types of data provide detail and comprehensive information about buildings, infrastructures and assets. Building information modelling (BIM) which is referred to as a process to help manage complexity and improve performance in construction projects throughout its life-cycle provides such information. The use of BIM as a software application gives users the following advantages; 3D visualisation, change management, construction simulation, data management, operation management, etc. Architectural data that came from the engineering sketches is rich data with class information, such as DWG/DXF and BIM (rvt/ifc). IFC is an international standard (ISO 16739) and data format for the sharing and exchange of information within the AEC industry, it was developed by the buildingSMART in the year 1994. These data formats are very important to generate spatial objects in (LoD3 - LoD4), such as a building model. The integration of BIM with other systems like GIS has the advantage of having BIM models in the correct location based on global coordinates system (GCS) which are evidents of the following: sun and energy studies, site coordination for utilities and clash detection. Also, data represented in the form of geospatial perspective and detailed building information are conspicuously developing infrastructure development and smart city application. Therefore, based on the aforementioned state, we recommend using BIM as a system to boost the building industry in Oman (and Qatar). Also, BIM could be a rich source of 3D data for surface and subsurface for the development of 3D SDI. Currently, the use of BIM for 3D SDI in Oman and Qatar is limited.

2D File Formats: 2D file format is a standard for encoding spatial data. These formats are managed by mapping agencies and Oman SDI. These file formats include various service layers, such as buildings, roads, networks, utility, topography, and hydrology. This data available in Oman SDI geodatabase and can be accessed in different formats such as Shapefile (shp), Autodesk AutoCAD (dxf/dwg) and Bentley MicroStation (dgn file).

CityGML and CityJSON data formats: These file formats are essential and normally used for developing a 3D city model. CityGML is an open data model and XML-based data exchange format. It is used for describing relevant urban and landscape objects along with spatial and non-spatial attributes, relations, and hierarchical structures in five levels of detail (LoD). CityGML can be found in the form of a flat file (*.gml or *.json) or database (schema). CityGML uses 3D primitive geometry to represent $3 \mathrm{D}$ objects.

\subsection{Standards}

Different standards and models can be used to establish 3D spatial objects and 3D city models in the upcoming 3D SDI. Determining the 3D spatial standard is an important step for surface and subsurface spatial objects. However, this task is complex, and some issues still need to further academic research such as 3D data, 2.5D, LoD, 3D building, 3D model, as 
highlighted by several researchers, including (Biljecki, 2017; Kolbe, 2008; Stoter, 2020). CityGML and CityJSON standards proposed to manage some of the challenges that face implementing the 3D City model (Ledoux et al., 2019). Both can be used to exchange, store, and represent $3 \mathrm{D}$ objects in multiscale and level of detail (LoD). In contrast, CityGML is an official standard supervised by Open Geospatial Consortium (OGC), while CityJSON is still not yet (Vitalis et al., 2020). Both can present spatial objects that can be found in the cities like buildings, roads, tunnels, and others. CityGML creates a hierarchy structure both for geometric and semantic information. In contrast, CityJSON uses JSON coding to create the objects in a simple data structure. There are various spatial applications for CityGML standard and CityJSON, such as solar potential estimation, flood risk assessment, and noise (Arroyo Ohori, 2018).

\subsection{SDI Data Services}

Within an SDI it is important to record information about available datasets via metadata in order to find relevant data. Although current Oman SDI development focuses on 2D, however, there is a need for 3D SDI data services and other related functions.

We have developed a prototype 3D SDI data services that able to be served as a 3D geospatial data hub for various agencies, governmental sectors and stakeholders in Oman. The service also known as a 3D geoportal provides a platform for the users to explore, search, share and download spatial datasets.

The 3D geoportal is developed to enhance the awareness about 3D data availability and improve access to geospatial information, at the same time facilitating data sharing among participating agencies. Figure 5 shows the developed 3D geoportal used to publish and explore 3D data such as city models. The city model datasets are based on the international standard OGC CityGML.

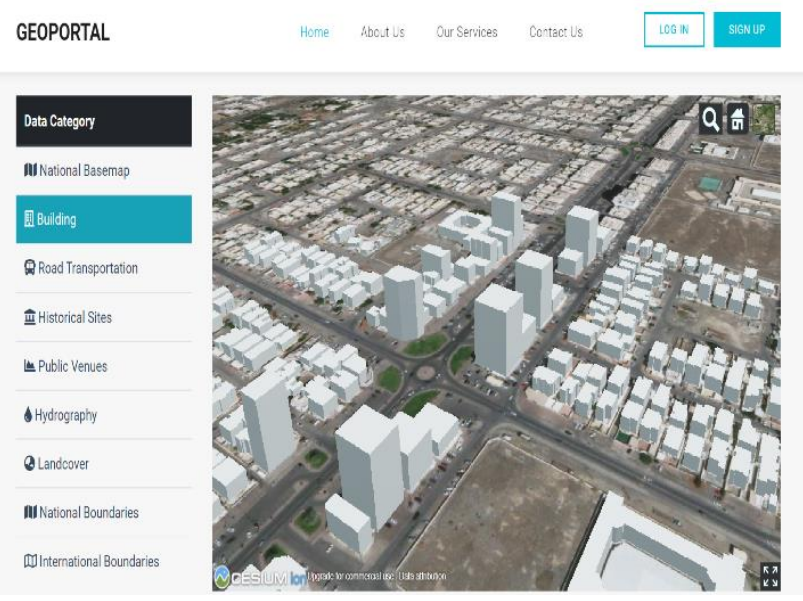

Figure 5. The developed 3D SDI data service for spatial data query, explore, share and download among various agencies in Oman.

Meanwhile, Figure 6 shows the explore function available in the geoportal, in which users can search for the specific dataset by selecting the sub-category (e.g. 3D building, footprint, wall and fences, etc.), governorate, wilayat (region), and town. Figure 7 shows windows based on the search results that show a list of available 3D data formats such as CityGML (LoD1 to LoD4), CityJSON, WFS, and BIM to be downloaded by the users.

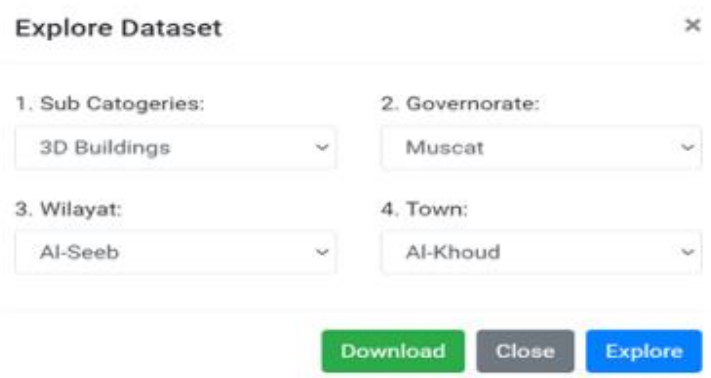

Figure 6. The explore function in the developed geoportal.

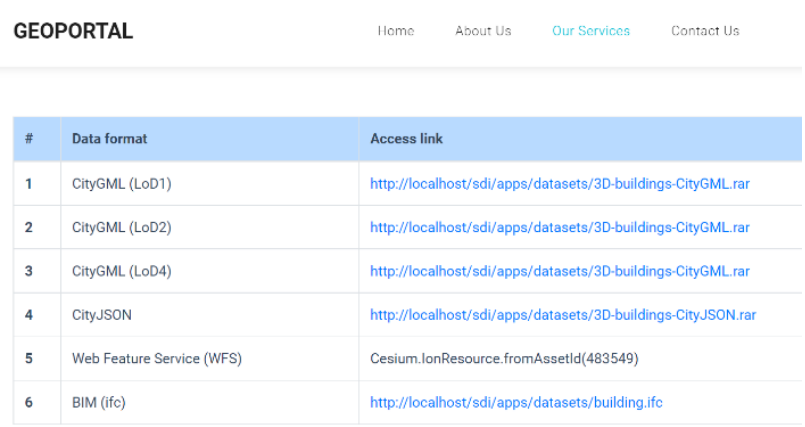

Figure 7. A list of available 3D data formats can be download from the geoportal.

The following Figure 8 shows the IFC model (LOD200) retrieved from the geoportal services within the "3D Buildings subcategory" (refer to Figure 6) comprises of some elements or components that constituted the IFC model such as IfcRoof, IfcWall, IfcSlab, etc. These elements can be used for applications like building management, cost estimation, building construction management, engineering services, fire and emergency services, and security (sensors).

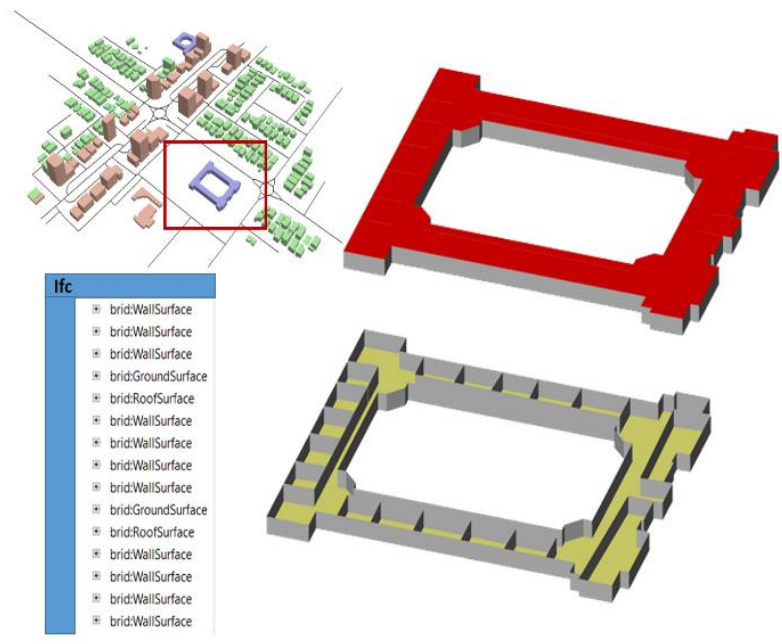

Figure 8: The IFC model of public school in Al-Khoud.

The proposed SDI data services will eventually create several applications by a specific agency such as a city municipality for 
flood risk assessment and airflow simulation in the urban area as depicted in Figure 9. Other useful applications such as shadow analysis, and solar radiation estimation could be developed.

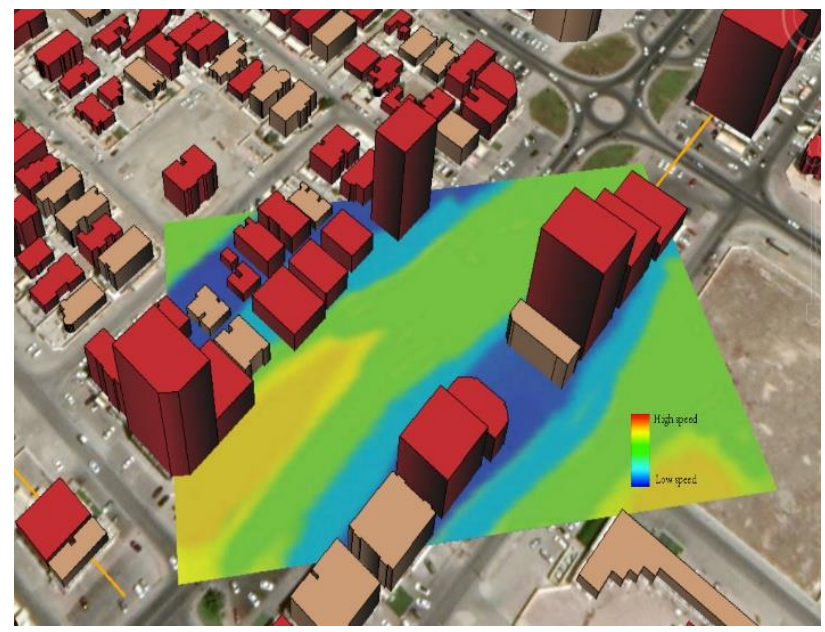

Figure 9: Airflow simulation in Al-Khoud.

The developed data service (refer to Figure 5) is just one of the components in the 3D SDI framework as shown in Figure 10. The framework consists of five major components, namely datasets and file formats, 3D geospatial database, interoperability platform, management system, and 3D visualisation system. These components are vital in the 3D SDI development in order to achieve a complete integrated platform.

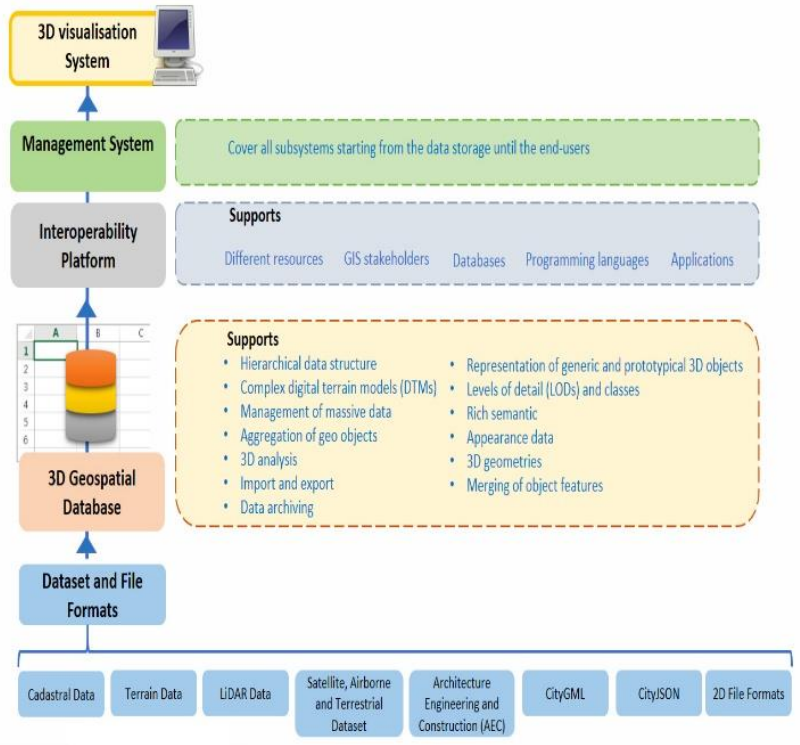

Figure 10. Proposed 3D SDI framework for Oman.

\section{ISSUES AND CHALLENGES}

Many SDI development initiatives face some challenges and issues ranging from working culture, policy to financial aspects. The willingness to invest in such national projects for SDI remains an issue (at least for the time being) for some countries. However, countries like Oman and Qatar seem not much have a problem to fully develop an SDI mainly due to available financial resources. The 2D SDI has gained quite significant outcomes where various stakeholders benefited from. However, migrating from $2 \mathrm{D}$ to $3 \mathrm{D}$ has some issues and challenges as highlighted in the following subsections.

\subsection{D spatial data availability}

There are various issues related to the 3D spatial data including the availability and quality. Our observation directs to a point where there is a need to improve the availability of 3D spatial data via developing mechanisms through the national SDI to maintain and sustain 3D spatial data in terms of modernity, quality, and timeliness. The authority may establish an initiative to acquire these kinds of spatial datasets since it has enormous potential for SDI development as proven by European-based SDIs. The developed SDIs has the 3D spatial data component as a based layer in their framework. A lot of investment has been allocated by their respective national agencies (e.g. Ordnance Survey UK, Dutch Kadaster Netherlands, IGN France). The same approach supposed to be established by the Oman SDI.

\subsection{D spatial data standards and data sharing}

Most of the 3D spatial data are structured and stored based on international standards such as CityGML and CityJSON. These standards are managed by ISO and OGC. The two data exchange standards are being used by many groups for the development of 3D SDI especially 3D city models. In this case, the Oman authority needs to update its standards to meet the requirement of 3D geospatial data. 3D city models based on CityGML and CityJSON require extra standards in terms of class, schema, geometric, semantic information, and also topology. All these possibilities of 3D spatial data standards need to be investigated and addressed for the 3D SDI development. Obviously, the data sharing also needs to be fully addressed by the authority. The national SDI should follow and adopt international best practices in this aspect.

\subsection{Related policy and laws}

It is very critical for the success of 3D SDI to have strong policies and rules that cover different aspects in the levels of $2 \mathrm{D}, 2.5 \mathrm{D}$, and 3D spatial data and the related services which cover procedures, best practices, and other authority directives. This is to formulate and promote 3D spatial data availability, data sharing, and other related issues with respect to data privacy and intellectual property. The lack of policies is a significant issue that needs to be discussed at the early stages.

\subsection{Technology and human capacity building}

SDI requires several GIS technologies related to data acquisition, data processing tools, database, data analysis, visualisation and publishing tools. Spatial data sharing tools (such as open-source and commercial GIS servers) play a big role in any SDI. Any new initiative on SDI has to be able to accommodate all types of 2D and 3D spatial data. Furthermore, the national agency needs to acquire the other important technologies as mentioned earlier (data processing tools, database, data analysis, visualisation, and publishing tools). On the other hand, the agency also needs to address human capacity building. The existing core skills for 3D SDI tasks within the agency are relatively low thus new schemes and opportunities for staff members to upgrade or acquire the right knowledge and skills are needed for managing the 3D SDI requirements (for the technical and administrative levels). 


\subsection{Applications for all social and economic sectors}

Ideally, national SDI agency should be able to serve all levels of users (public and private) in such a way that the available data is applicable for many spatial related applications such as for urban and rural planning, real estate, constructions and urban management, natural resources and disaster management, transportation and aviation, telecommunication and utility (electricity, oil and gas, water, sewerage), health, meteorology, education, banking and finance, and homeland security. One of the ways to overcome the spatial data sharing and services between stakeholders and users via a flexible online geospatial service platform. We anticipate this kind of service inevitably create a new geospatial paradigm for the national agency.

\section{CONCLUSION}

This paper discussed the spatial data infrastructure (SDI) of Oman and Qatar - current status and perspective. It also shows that the 3D SDI is one of the important initiatives for the nation's development. Currently, the two countries achieved some progress on the subject; however, there are several issues and challenges that need to be addressed as discussed in the previous sections. In the near future, based on the outcome of the works, we would like to explore new solutions for all stakeholders and users in Oman and Qatar. We believe that this piece of research idea will significantly trigger the importance of $3 \mathrm{D}$ spatial solutions for national sustainable development such as the sustainable smart city.

\section{ACKNOWLEDGEMENTS}

The authors would like to acknowledge the Ministry of Higher Education, Research, and Innovation (MoHERI) of the Sultanate of Oman for supporting the main project (3D SDI).

\section{REFERENCES}

Abbas, R., Behnam, A., \& Mohsen, K. (2019). BIM and Urban Land Administration. Boca Raton : Taylor \& Francis, CRC Press, 2019.: CRC Press. https://doi.org/10.1201/9781351032346

Arroyo Ohori, K., Biljecki, F., Kumar, K., Ledoux, H., \& Stoter, J. (2018). Modeling Cities and Landscapes in 3D with CityGML. In Building Information Modeling (pp. 199-215). Cham: Springer International Publishing. https://doi.org/10.1007/978-3319-92862-3_11

Biljecki, F. (2017). Level of Details in 3D City Models, Published $\mathrm{PhD}$ Thesis. Delft University of Technology. https://doi.org/10.4233/uuid:f12931b7-5113-47ef-bfd4688aae3be248

Cheoung, P. (2011). Keynote Talk on Status of SDI of Member States. UN GGIM Meeting, Seoul, Korea.

Chaturvedi, K., \& Kolbe, T. (2019). Towards Establishing CrossPlatform Interoperability for Sensors in Smart Cities. Sensors, 19(3), 562. https://doi.org/10.3390/s19030562

Das, A., Chandel, K., \& Narain, A. (2017). Value of Geospatial Technology in Boosting Omans Economy. In Oman Geospatial Forum 2017 (pp. 1-74). Muscat: Oman National Survey Authority.
Kolbe, T. H. (2008). Representing and Exchanging 3D City Models with CityGML. 3D Geo-Information Sciences, 15-31. https://doi.org/10.1007/978-3-540-87395-2_2

Ledoux, H., Arroyo Ohori, K., Kumar, K., Dukai, B., Labetski, A., \& Vitalis, S. (2019). CityJSON: A Compact and Easy-to-Use Encoding of the CityGML Data Model. Open Geospatial Data, Software and Standards, 4(1), 1-19. https://doi.org/10.1186/s40965-019-0064-0

NCSI. (2017a). Oman National Spatial Data Infrastructure (1st ed.). Oman: National Center for Statistics and Information. Retrieved from https://www.ncsi.gov.om/Elibrary/LibraryContentDoc/ben_Om an National Spatial Data Infrastructure_1303dfbd-ce6d-4e13afee-62c137632eef.pdf

NCSI. (2017b). Oman National Spatial Data Infrastructure Strategy V5.0. Oman: National Center for Statistics and Information. Retrieved from https://www.ncsi.gov.om/Elibrary/LibraryContentDoc/ben_Om an National Spatial Data Infrastructure_1303dfbd-ce6d-4e13afee-62c137632eef.pdf

Stoter, J. (2020). State of the Art in 3D City Modelling - Six Challenges Facing 3D Data as a Platform. GIM International, 47.

UN GGIM (2011). Plenary session at the UN GGIM Meeting in Seoul, Korea

UN GGIM (2013). Plenary session at the UN GGIM Meeting in Doha, Qatar.

Vitalis, S., Labetski, A., Boersma, F., Dahle, F., Li, X., Arroyo Ohori, K., ... Stoter, J. (2020). CITYJSON + WEB = NINJA. ISPRS Annals of Photogrammetry, Remote Sensing and Spatial Information Sciences, VI-4/WI-20, 167-173. https://doi.org/10.5194/isprs-annals-VI-4-W1-2020-167-2020 